\title{
El color en los códices prehispánicos del México Central: identificación material, cualidad plástica y valor estético
}

\author{
Élodie DuPEY GARCÍA \\ Instituto de Investigaciones Históricas, Universidad Nacional Autónoma de México \\ edupeygarcia@gmail.com
}

Recibido: 10 de octubre de 2014

Aceptado: 15 de noviembre de 2014

\begin{abstract}
RESUMEN
Este artículo investiga algunos de los valores plásticos y estéticos que presidieron la selección y la preparación de las materias colorantes empleadas para iluminar los códices creados por los nahuas del México Central durante el Posclásico Tardío. Estos códices son interesantes porque análisis arqueométricos y exámenes codicológicos recientes han permitido conocer la materialidad de su capa pictórica, así como las características formales y el comportamiento de los colores en estas obras. Uno de los aportes trascendentales de estos estudios ha sido averiguar que la paleta cromática que sirvió para pintar los códices del México Central era principalmente de origen orgánico, lo que contrasta con la naturaleza de los pigmentos detectados en restos de pintura mural y en esculturas creadas por los nahuas que son sobre todo minerales. El objetivo de este artículo es reflexionar sobre las razones de esas diferencias y demostrar que el uso de los colorantes orgánicos en los códices respondía a un fin plástico específico que concordaba con el canon estético imperante en la sociedad náhuatl.
\end{abstract}

Palabras clave: Códices del Grupo Borgia, pigmentos, colorantes, valores plásticos, canon estético.

\section{The Colors of the Pre-Hispanic Codices from Central Mexico: Material Characterization, Plastic Qualities and Aesthetic Values}

\begin{abstract}
This article outlines the investigation of some of the plastic and aesthetic values that presided over the selection and preparation of the colorant materials employed to illuminate the codices created by the Nahua of Central Mexico during the Late Postclassic. These codices are interesting as recent archaeometrical analysis and codicological examination has revealed the materiality of their pictorial layers together with the formal characteristics and behavior of colors in these works of art. One of the most significant contributions of these studies has undoubtedly been the discovery that the chromatic palette employed in the painting of the codices of Central Mexico was primarily of organic origin. This being in stark contrast to the nature of the pigments detected in the remains of mural paintings and in the sculptures created by the Nahua, which were mostly of mineral origin. The objective of this article is to examine the reason for these differences and to demonstrate that the use of pigments prepared with organic colorants in the codices obey to a specific plastic purpose that coincided with the prevailing aesthetic ideals of Nahuatl society.
\end{abstract}

Key words: Borgia Group codices, pigments, dyes, plastic values, aesthetic canon.

Sumario: 1. Introducción, 2. La paleta cromática de los códices prehispánicos del México Central. 3. Cualidad plástica y valor estético del color en los códices. 4. Conclusiones. 5. Referencias bibliográficas.

\section{Introducción}

En este ensayo se presenta la investigación de algunos de los valores plásticos y estéticos que presidieron a la selección y la preparación de las materias colorantes empleadas para iluminar los códices creados en el México Central durante el Posclásico Tardío (1200-1521 d.C.). Concretamente, el corpus considerado reúne algunos 
de los códices del llamado Grupo Borgia ${ }^{1}$ : Borgia, Cospi, Fejérváry-Mayer, Laud y Vaticano $B 3773^{2}$. Más de un siglo de investigación ha demostrado que pese a su origen desconocido y sus diferencias estilísticas, estos códices mantuvieron estrechas relaciones con la cultura náhuatl que prosperó en el Centro de México durante el Posclásico Tardío. Comparaciones con murales y piezas de cerámica polícromas sugieren incluso que los códices Borgia y Cospi (anverso) fueron pintados en el Valle de Puebla-Tlaxcala, mientras que el Códice Laud pudo provenir del Valle de Tehuacán (Caso 1927; Contreras 1994; Boone 2007; Álvarez 2014).

Estos códices son interesantes también porque análisis arqueométricos y exámenes codicológicos ${ }^{3}$ recientes han permitido conocer la materialidad de su capa pictórica, así como las características formales y el comportamiento de los colores en estas obras. En los últimos siete años, he realizado el estudio codicológico de los códices Borgia, Cospi, Laud y Vaticano B. En cada ocasión observé la integralidad de las láminas con detenimiento, lo cual me ha permitido conocer la paleta de colores de cada documento, así como advertir las similitudes y diferencias cromáticas que tienen entre sí o que aparecen en el seno de un mismo manuscrito. Paralelamente, los manuscritos del Grupo Borgia han sido examinados con técnicas arqueométricas no invasivas por un equipo de químicos llamado $\mathrm{MOLAB}^{4}$, con el fin de identificar sus materiales constitutivos, en particular los colores que sirvieron para pintarlos. A la fecha, este equipo ha dado a conocer los resultados de los estudios de los códices Cospi y Fejérváry-Mayer (Miliani et al. 2012; Domenici et al. 2014).

Sin duda, uno de los aportes trascendentales de estos análisis arqueométricos -aunados a los resultados de estudios similares que se han efectuado en manuscritos procedentes de la Mixteca- ha sido averiguar que la paleta cromática que sirvió para pintar los códices del México Central era principalmente de origen orgánico. Este descubrimiento contrasta con la naturaleza de los colores detectados en restos de pintura mural y en esculturas creadas por los nahuas - procedentes, en concreto, de la antigua ciudad de Tenochtitlan- los cuales son sobre todo minerales. Mi objetivo en

1 El concepto de Grupo Borgia fue creado por Eduard Seler $(1963,1990)$ y es generalmente aceptado aunque ha dado lugar a importantes discusiones. En efecto, si bien desde el punto de vista temático la agrupación de Seler parece correcta porque los códices que conforman el grupo tratan de temas semejantes que giran en torno a cuestiones religiosas, a nivel estilístico se observa que aunque existen cualidades comunes a estos documentos, los códices del llamado Grupo Borgia no comparten un estilo común ni monolítico. Sobre este tema, véanse por ejemplo: Boone 1990; 2007; Escalante Gonzalbo 1996; 2010; Batalla 2008a: 247-261; Dupey García y Álvarez Icaza, en prensa.

2 Aunque los códices Porfirio Díaz (reverso) y Fonds Mexicain 20 se incluyen a veces en el Grupo Borgia, no los consideramos en este trabajo debido a que el reverso del primero no es polícromo, mientras que la capa pictórica del segundo está muy deteriorada y no permite el análisis de sus características cromáticas.

$3 \mathrm{La}$ arqueometría es una disciplina que emplea métodos físico-químicos para estudiar bienes culturales y sitios arqueológicos e históricos. Sus objetivos principales son la datación de dichos bienes y vestigios, la identificación de la tecnología empleada para elaborarlos, así como la caracterización de sus materiales constitutivos (origen, propiedades físicas y químicas, etc.). La codicología es la disciplina que estudia los manuscritos como objetos físicos, para averiguar su proceso de creación. En el caso de los códices prehispánicos informa en especial sobre la confección del soporte y la técnica de aplicación de los colores, etc. (véase, por ejemplo: Batalla 2008b: 45-48).

4 El MOLAB es un órgano del Center of Excellence SMAArt y del CNR-ISTM de Perugia. Los químicos que conforman este equipo trabajan en colaboración con Davide Domenici, investigador de la Universidad de Bolonia. 
este trabajo es reflexionar sobre la razón de ser de tal diferencia, así como demostrar que la aplicación de colores cuyo principio colorante era orgánico en los códices respondía a un fin plástico específico que concordaba con el canon estético imperante en la sociedad náhuatl.

Para ello, la presente investigación descansa en un cotejo de los datos codicológicos y arqueométricos acerca de la materialidad del color en los códices con los testimonios históricos. La arqueometría, en efecto, caracteriza los materiales colorantes empleados. La codicología proporciona noticias respecto de las técnicas y estrategias artísticas utilizadas para elaborar estos manuscritos, a la vez que informa sobre las preferencias de los nahuas en materia de colores. La documentación histórica, finalmente, recoge la forma de nombrar y describir tintes y pigmentos entre los nahuas, así como discursos nativos sobre la creación artística y las cualidades de ciertos materiales colorantes.

\section{La paleta cromática de los códices prehispánicos del México Central}

El soporte de los manuscritos del Grupo Borgia -y de los códices prehispánicos procedentes de la Mixteca- es piel animal. Después de ser sometida a un proceso de preparación que incluía limpieza, depilación y curtido (Maldonado y Maldonado 2004; Zetina et al. 2014), la piel recibía la aplicación de un enlucido blanco que se conoce como «base de preparación», porque sirve de base firme y homogénea para la capa pictórica ${ }^{5}$.

Con excepción de este enlucido blanco, que era sistemáticamente de origen mineral, los colores de estos códices eran, por lo general, de origen orgánico (Cuadro 1), según han revelado los análisis arqueométricos efectuados sobre los códices Cospi y Fejérváry-Mayer (Miliani et al. 2012; Domenici et al. 2014) al igual que sobre códices mixtecos (Strebinger 1961; Torres et al. 1966; Zetina et al. 2011, 2014; Higgitt 2013). Así, los únicos minerales detectados en estos documentos son el sulfato de calcio $\left(\mathrm{CaSO}_{4} \cdot 2 \mathrm{H}_{2} \mathrm{O}\right)$ y la anhidrita $(\mathrm{CaSO})$ usados para preparar la base de preparación -que hacía también las veces de blanco- y el oropimente $\left(\mathrm{As}_{2} \mathrm{~S}_{3}\right)$ que sirvió para pintar ciertas zonas de amarillo (Miliani et al. 2012: 675-678; Domenici et al. 2014: 107-108) ${ }^{6}$. Aparte de estos materiales inorgánicos, los demás colores aplicados en estos códices eran preparados a partir de materias colorantes de origen animal o vegetal; una observación que coincide con un pasaje de la obra de Motolinía (1971: 218), donde el fraile franciscano explica que «muchas colores hacen los indios de flores, y cuando los pintores quieren mudar el pincel de una color en otra, con la boca limpian el pincel, por ser las colores de flores» ${ }^{7}$.

5 Sobre la base de preparación de los códices del Grupo Borgia, véanse: Laurencich et al. 1993; Miliani et al. 2012: 674-677; Domenici et al. 2014: 101-104.

6 El oropimente fue empleado también en el Códice Zouche-Nuttall y en códices coloniales como el Florentino y Badiano (Zetina et al. 2008; Baglioni et al. 2011: 100; Higgitt 2013).

7 Un comentario análogo se encuentra bajo la pluma del dominico Diego de Landa (1994: 213), quien escribe acerca de la tierra de Yucatán que: «Colores hay de muchas diversidades, hechos de tintas de algunos árboles, y de flores». 
Cuadro 1: Materias colorantes identificadas en códices del México Central mediante técnicas arqueométricas y materias colorantes descritas en las fuentes históricas y susceptibles de haber sido utilizadas en los códices

\begin{tabular}{|c|c|c|}
\hline Colores & $\begin{array}{c}\text { Materias colorantes } \\
\text { identificadas } \\
\text { arqueométricamente }\end{array}$ & Materias colorantes descritas en las fuentes históricas \\
\hline Blanco & $\begin{array}{l}\text { Sulfato de calcio } \\
\text { Anhidrita }\end{array}$ & $\begin{array}{l}\text { Tetizatl: sulfato de calcio o carbonato de calcio } \\
\text { Chimaltizatl: selenita }\end{array}$ \\
\hline Negro & Negro de humo & Tlilli o tlilliocotl: negro de humo \\
\hline Rojo & Grana cochinilla & $\begin{array}{l}\text { Tlacuahuac tlapalli: pigmento laca rojo de grana cochinilla } \\
\text { Camopalli: pigmento laca rojo oscuro de grana cochinilla } \\
\text { Achiyotl: pigmento laca rojo a base de achiote } \\
\text { Huitzcuahuitl: palo Brasil }\end{array}$ \\
\hline Amarillo & Oropimente & $\begin{array}{l}\text { Zacatlaxcalli: pigmento laca amarillo de Cuscuta } \\
\text { Xochipalli: pigmento laca amarillo de Cosmos sulphureus } \\
\text { Tecozahuitl: pigmentos laca amarillos preparados con arcilla }\end{array}$ \\
\hline Azul & Azul maya & $\begin{array}{l}\text { Texotli: azul maya y pigmentos laca azules preparados con arcilla } \\
\text { Tlacehuilli: índigo } \\
\text { Matlalli: pigmento laca azul de Commelina coelestis }\end{array}$ \\
\hline Verde & $\begin{array}{l}\text { Mezcla de azul maya } \\
\text { con un tinte amarillo }\end{array}$ & $\begin{array}{l}\text { Quiltic: mezcla de texotli con zacatlaxcalli } \\
\text { Yappalli: mezcla de texotli con zacatlaxcalli }\end{array}$ \\
\hline
\end{tabular}

Las fuentes históricas y los estudios científicos coinciden en que los colores de origen orgánico usados en los códices podían ser colorantes que se extraían, se depuraban, se mezclaban con algún aglutinante y, sin requerir más preparación, se utilizaban para pintar. Sin embargo, la mayoría de los materiales colorantes orgánicos eran convertidos en pigmentos laca para alcanzar la estabilidad y la resistencia que su aplicación en los códices exigía. Los pigmentos laca mesoamericanos se obtenían precipitando uno o varios componentes orgánicos sobre un sustrato mineral (Dark y Plesters 1959; Falcón 2014: 9-15), que puede ser una sal metálica -alumbre $\left(\mathrm{KAl}\left(\mathrm{SO}_{4}\right)_{2} \cdot 12 \mathrm{H}_{2} \mathrm{O}\right)$, nitro $\left(\mathrm{KNO}_{3}\right)$ o aceche $\left(\mathrm{FeSO}_{4}\right)$, o un tipo específico de arcilla. La preparación de estos pigmentos recuerda entonces el proceso tintóreo, porque implicaba el calentamiento de las materias orgánicas e inorgánicas para que el tinte se fijara sobre las partículas del sustrato inerte (Roquero 2006: 89-90, 100-101; Falcón 2014; Zetina et al. 2014: 137). El precipitado resultante era filtrado y se dejaba secar, convirtiéndose entonces en color para pintar.

Entre los colores de origen vegetal utilizados en los códices destaca el negro humo, cuya presencia se detectó men los códices del Grupo Borgia analizados mediante técnicas arqueométricas: el Cospi y el Fejérváry-Mayer (Miliani et al. 2012: 675; Domenici et al. 2014: 104) ${ }^{8}$. La documentación histórica corrobora el uso de este material a base de de carbón vegetal para delinear y colorear, a la vez que proporciona su nombre en náhuatl: tlilli o tlilliocotl (Sahagún 1953-1982 X: 28, XI: 242-243; 2000: Libro X, capítulo 8: 875; Dupey 2015: 254). Los textos coloniales también explican la técnica que emplean los indígenas para conseguir esta materia colorante:

«[...] con humo de astillas de cualquier pino (ócotl en la lengua vernácula y de donde la tinta toma el nombre), que dentro de vasijas cerradas se condensa en pelotillas, las

8 Es lo mismo en los demás manuscritos prehispánicos, mixtecos y mayas (Strebinger 1961: 23; Torres et al. 1966: 92; Zetina et al. 2011: 352-353; 2014; 132, 135-136; Buti et al. 2014: 175). 
cuales arrancadas luego se venden con frecuencia en los mercados» (Hernández 1959 III: 409).

En cuanto a los rojos de los códices del Grupo Borgia fueron elaborados con un colorante orgánico, probablemente de origen animal, cuyo comportamiento ante los estudios arqueométricos es similar al de la grana cochinilla (Miliani et al. 2012: 675, 677; Domenici et al. 2014: 105-106) ${ }^{9}$. En el anverso del Cospi, la grana cochinilla aparece mezclada con otro rojo orgánico que no se ha podido identificar, razón por la cual es interesante recurrir a las fuentes históricas para formular algunas hipótesis.

En la documentación colonial -especialmente en el Códice Florentino de Bernardino de Sahagún (1953-1982 XI: 239-240, 242; Dupey 2015: 245-248, 252, 258)- se describen las virtudes colorantes de la grana cochinilla, a la vez que se facilitan los nombres vernáculos de la grana en bruto (nocheztli), así como de los tintes y pigmentos laca que se derivaban de este material. Entre ellos, destaca el tlacuahuac tlapalli o «tlapalli duro» que era un pigmento laca elaborado con grana cochinilla -asociada con alumbre, aceche y las hojas de la planta tezuatl ${ }^{10}$ - y empleado para pintar los códices (Sahagún 1953-1982 XI: 242; Hernández 1959 II: 315; Falcón 2014: 27-36; Dupey 2015: 247-248, 252). Se menciona asimismo un pigmento laca rojo oscuro tirando a morado llamado camopalli -literalmente «el tinte [color] de camote»-que se preparaba con grana cochinilla mezclada con alumbre (Sahagún 1953-1982 XI: 244-245; 1979 III: 372v; 2000: Libro XI, capítulo 11: 1133; Dupey 2015: 258).

Los textos coloniales citan también un pigmento laca llamado achiyotl en náhuatl, elaborado con achiote (Bixa orellana) y utilizado para pintar los códices (Sahagún 1953-1982 XI: 241; Hernández 1959 II: 27-28; Dupey 2015: 249-250). Señalan, además, que el achiote se mezclaba con grana para preparar un color rojo (Motolinía 1971: 218), lo que invita a pensar que el anverso del Cospi pudo ser pintado precisamente con esta mezcla de grana y achiote. No se debe descartar, sin embargo, que dicho color se haya preparado mezclando grana con el colorante extraído de la madera del Haematoxylon brasiletto, un árbol que pertenece a la familia del palo Brasil y que los nahuas nombraban huitzcuahuitl, «árbol de espinas» (Roquero 2006: 119-129). Los textos históricos revelan que en la época prehispánica se obtenía de este vegetal «un color para los pintores [cuyo] rojo [era] más bello que el cinabrio» (Hernández 1959 II: 337, 375; 1998 I: 380-381).

Con el amarillo, el naranja y el café, las gamas cromáticas de los manuscritos del México Central se vuelven más complejas. Como se indicó, los códices Cospi y Fejérváry-Mayer incluyen un amarillo especialmente brillante preparado con oropimente. Además, las paletas de estos manuscritos incluyen pigmentos laca-cuyos matices van del amarillo al café- obtenidos a partir de diferentes colorantes, algunos

9 Este material se obtiene del cuerpo de las hembras del insecto Dactylopius coccus que vive sobre las pencas del nopal (Opuntia spp.). En los códices coloniales se ha identificado grana cochinilla, ocasionalmente precipitada sobre una arcilla, pero también materiales inorgánicos como la hematita $\left(\mathrm{Fe}_{2} \mathrm{O}_{3}\right)$, el minio $\left(\mathrm{Pb}_{3} \mathrm{O}_{4}\right.$ o $2 \mathrm{PbO} \cdot \mathrm{PbO}_{2}$ ) y el cinabrio $(\mathrm{HgS})$, a veces mezclados entre sí o con colorantes para obtener nuevos tonos y matices (Dark y Plesters 1959: 532, 537; González 1998: 27; Haude 1998; Baglioni et al. 2011: 87-94; Magaloni 2011: 66-67; Zetina et al. 2011: 353).

10 Las hojas del tezuatl (Miconia sp.) contienen aluminio y actúan como mordiente vegetal (Roquero 2006: 94-95, 103, 140; Falcón 2014: 27-36). 
de ellos fijados sobre arcillas (Miliani et al. 2012: 675-678; Domenici et al. 2014: 101, 107-108). Pigmentos laca amarillos y anaranjados se encontraron también en los códices mixtecos Zouche-Nuttall y Colombino-Becker I (Torres et al. 1966: 9293; Zetina et al. 2011: 352; 2014: 137; Higgitt 2013) y en los manuscritos coloniales Selden y Florentino (Dark y Plesters 1959: 533, 538; Baglioni et al. 2011: 98-100) ${ }^{11}$.

Por el momento, las materias orgánicas en el origen de estos pigmentos laca no han sido identificadas mediante técnicas arqueométricas, pero las fuentes históricas revelan que dos pigmentos laca preparados con sales metálicas y colorantes vegetales servían para pintar de amarillo en los códices nahuas: el zacatlaxcalli y el xochipalli. Zacatlaxcalli era el nombre que los nahuas atribuían a un pigmento laca con un intenso color amarillo y de origen carotenoide, obtenido de varias especies de plantas parásitas del género Cuscuta maceradas con alumbre y nitro. La palabra xochipalli designaba un colorante y un pigmento laca de matiz amarillo anaranjado elaborados con las flores del cosmos (Cosmos sulphureus) -cuyo principio colorante es un flavonoide- cocidas con nitro (Hernández 1959 II:124, 3:212-213; Sahagún 1953-1982 XI: 240; 1979 III: 369r, 369v; Wallert 1995: 657-659; Falcón 2014: 37-39, 43-45; Dupey 2015: 248-249) ${ }^{12}$.

Paralelamente, la documentación histórica sugiere que el léxico náhuatl incluía un término -tecozahuitl- que designaba los pigmentos laca elaborados precipitando tintes amarillos sobre arcillas, como los que se han detectado en los códices Cospi y Fejérváry-Mayer ${ }^{13}$. Los colaboradores de Sahagún (1953-1982 XI: 242; 1979 III: 371r; Dupey 2015: 253) describen y representan al tecozahuitl como una piedra amarilla que sirve para teñir y pintar, mientras Francisco Hernández (1959 III: 410) lo define como «una especie de ocre o tierra amarilla [...] con que los pintores dan dicho color» y que «favorece el cutis agrietado». Este último dato apunta a que una arcilla entraba en la composición del tecozahuitl porque las virtudes hidratantes de estas tierras son reconocidas. Andrés de Olmos (2004 I: s. v. tecoçahuitl), por su parte, traduce tecozahuitl como «yerva amarilla» ${ }^{14}$. Esta naturaleza a la vez mineral y vegetal del tecozahuitl apunta a que éste era el nombre que los nahuas atribuían a los pigmentos laca obtenidos a partir de colorantes amarillos y arcillas (Dupey 2010: 83-85).

Respecto de los azules y verdes, se han identificado colores de composición diferente en los códices Cospi y Fejérváry-Mayer. Así, el azul verdoso del anverso del

11 Cabe mencionar que el Códice Selden presenta una composición física y un estilo totalmente prehispánicos (Batalla 2008a: 203-204).

12 La posibilidad de que los pigmentos de color amarillo-anarajando y amarillo-dorado que se observan en códices prehispánicos y coloniales hayan sido preparados respectivamente con Cosmos sulphureus (xochipalli) y Cuscuta (zacatlaxcalli) ha sido formulada también por Dark y Plesters (1959: 533) y Domenici et al. (2014: 108).

13 En cambio, no se ha encontrado en la documentación colonial la descripción de un mineral con las características del oropimente.

14 Además, el Códice Florentino de Sahagún (1953-1982 XI: 245; 2000: Libro XI, capítulo 11: 1133; Dupey 2015: 259-260) menciona la existencia de un vínculo entre el tinte amarillo tirando a café llamado cuappachtli y el pigmento tecoxtli, cuyo nombre es una variante de tecozahuitl y significa también «piedra amarilla». Los informantes de Sahagún describen al cuappachtli como un liquen -posiblemente del género Dolichocarpus (Torres 2001: 347; Roquero 2006: 191-192)- utilizado para teñir de amarillo oscuro o de café, mientras que el franciscano indica que el color cuappachtli se deriva de la mezcla de la piedra tecoxtli con el aglutinante orgánico que se solía usar para vehicular pigmentos en la época prehispánica. 
Cospi combina un tinte azul con un pigmento laca amarillo; en tanto que los azules de matiz turquesa que se observan en el Fejérváry-Mayer y en el reverso del Cospi fueron pintados con azul maya (Miliani et al. 2012: 676-677; Domenici et al. 2014: $110)^{15}$. El azul maya es un pigmento laca que resulta de la fijación del índigo obtenido de la planta de añil (Indigofera suffruticosa) sobre arcillas blancas, en particular la palygorskita $\left((\mathrm{Mg}, \mathrm{Al})_{2} \mathrm{Si}_{4} \mathrm{O}_{10}(\mathrm{OH}) \cdot 4\left(\mathrm{H}_{2} \mathrm{O}\right)\right)^{16}$. Se ha afirmado que este pigmento laca no se menciona en las fuentes históricas (Reyes 1993), y es cierto que los autores del siglo XVI no proporcionan la receta para prepararlo, ni describen una arcilla que tenga las características de la palygorskita.

La planta de añil-xiuhquilitl en náhuatl- requerida para preparar el azul maya, en cambio, es descrita en la documentación colonial, que facilita asimismo el nombre que los nahuas daban al índigo extraído de sus hojas: tlacehuilli. Esta documentación revela además que el tlacehuilli se usaba para pintar códices y que su color era llamado mohuitic en náhuatl, esto es, azul oscuro tirando a violeta (Hernández 1959 III: 112-113; Sahagún 1953-1982 XI: 242; 2000: Libro XI, capítulo 11: 1131; Dupey 2010: 284-286; 2015: 252-253). A la fecha, no se ha detectado el uso de tlacehuilli, es decir de índigo, para pintar de azul oscuro en códices prehispánicos, pero sabemos que fue aplicado en códices coloniales (González 1998: 27, 89; Haude 1998; Baglioni et al. 2011: 97).

Aparte del índigo, los textos históricos citan otro material utilizado para pintar de azul: el texotli. Planteo que éste pudo ser el nombre náhuatl de la categoría de pigmentos laca que se obtenían precipitando un colorante azul sobre una arcilla, entre ellos el azul maya. Los informantes de Sahagún (1953-1982 XI: 242; Dupey 2015: 253) definen al texotli como un material de color turquesa intenso, como el azul maya, mientras el franciscano piensa que tenía un origen orgánico y sostiene que se producía con las flores matlalxochitl (Commelina coelestis) (Sahagún 2000: Libro XI, capítulo 11: 1132) ${ }^{17}$. Hernández (1959 III: 407), por su parte, identifica el texotli con un mineral, lo que coincide con su nombre de «piedra azul». Como en el caso del tecozahuitl, reconciliar estos puntos de vista es posible si consideramos que texotli era el nombre que se daba, en náhuatl, al pigmento laca preparado con una arcilla y las flores matlalxochitl, así como a todos los pigmentos laca-incluido el azul mayaobtenidos precipitando un tinte azul sobre una arcilla (Dupey 2010: 83-85) ${ }^{18}$.

15 Este pigmento laca se usó también en los códices mixtecos Zouche-Nuttall y Colombino, así como en documentos coloniales (Torres et al. 1966: 93; Haude 1998; Higgitt 2013; Zetina et al. 2014: 135).

16 La historia de la investigación dedicada al azul maya es larga, al igual que la lista de publicaciones que tratan sobre este pigmento. Síntesis sobre estas cuestiones se pueden encontrar en: Reyes 1993; Doménech et al. 2014.

17 Las flores matlalxochitl también servían para preparar el color matlalli, que se usaba para pintar códices y bien pudo ser un pigmento laca preparado con sales metálicas (Falcón 2014: 39; Dupey 2015: 249).

18 Por derivación, es posible que la palabra texotli haya sido utilizada, en la época colonial, para nombrar pigmentos inorgánicos azules, como la azurita $\left(\mathrm{Cu}_{3}\left(\mathrm{CO}_{3}\right)_{2}(\mathrm{OH})_{2}\right)$. En efecto, Falcón (2014: 61-62) señala que la descripción del texotli por Hernández se refiere al procedimiento empleado en el Antiguo Mundo para obtener azurita mediante lavados sucesivos y filtrado. Sin embargo, de acuerdo con los conocimientos actuales, la azurita es un pigmento ausente de la paleta de los pintores de códices en el Centro de México durante el Posclásico. En consecuencia, la utilización de la palabra texotli para designar la azurita en la obra de Hernández podría ser una creación colonial, probablemente ocasionada por la ignorancia que tenían los españoles de los pigmentos laca obtenidos fijando tintes azules sobre arcillas. 
El verde es un color de sorprendente variedad en los códices del Grupo Borgia. El análisis arqueométrico del Códice Fejérváry-Mayer ha revelado que uno de sus verdes se derivaba de la mezcla de azul maya con materiales colorantes amarillos que permanecen sin identificar (Domenici et al. 2014: 110). Mi estudio codicológico del Cospi me permitió observar que el verde del anverso de este códice, en cambio, se origina por la superposición de una capa de amarillo sobre una de azul, o viceversa. Esta técnica que consiste en sobreponer capas de color para obtener matices verdes ocurre también en dos códices mixtecos: el Colombino y el Selden. En estas obras, el verde resulta de la aplicación de capas sucesivas de materiales amarillo y azul, o negro (Dark y Plesters 1959: 533; Torres et al. 1966: 96; Zetina et al. 2014: 130-132).

Las fuentes históricas no evocan la posibilidad de crear el color verde por medio de capas. Más bien, el capítulo sobre colores del Códice Florentino describe el proceso de fabricación de dos verdes mediante la mezcla de los mismos materiales amarillo y azul: el zacatlaxcalli y el texotli. (Sahagún 1953-1982 XI: 244-245; Dupey 2015: 257-258). Uno de estos verdes se llamaba quiltic, un término común para designar el verde en náhuatl que significa «como una verdura» (Dupey 2010: 277-280). Éste es descrito por los informantes de Sahagún como «un poco azul-verde, un poco amarillo oscuro». El otro material verde se llamaba yappalli, una palabra que significa «tinte de maíz negro» porque combina el verbo $p a$, «teñir», con el sustantivo yahuitl, «mazorca de maíz negro» (Dupey 2015: 257). Sahagún (2000, Libro XI, capítulo 11: 1133) sostiene que el yappalli es un «verde escuro», en tanto que sus informantes indican que: «No es completamente azul-verde. No es amarillo. El amarillo que se deriva de [esta mezcla] es muy oscuro» (Dupey 2015: 257).

Estas descripciones son dignas de atención porque los ingredientes citados recuerdan la composición de uno de los colores verdes aplicados en el Códice FejérváryMayer. Davide Domenici et al. (2014: 110) indican, en efecto, que se trata de una mezcla de azul maya con un material amarillo que no se ha identificado, pero que pudo ser el zacatlaxcalli dado que este pigmento laca destaca en los testimonios históricos que se refieren a la paleta de los pintores de códices. En cuanto al azul maya era uno de los materiales básicos para la producción del color verde ${ }^{19}$. Resulta significativo, entonces, encontrar al texotli entre los ingredientes requeridos para elaborar los verdes quiltic y yappalli, ya que he formulado la hipótesis de que texotli fue el nombre náhuatl de la categoría de pigmentos laca preparados fijando un tinte azul sobre una arcilla, entre ellos el azul maya.

\section{Cualidad plástica y valor estético del color en los códices}

Como hemos visto, las fuentes históricas más relevantes para investigar los colores de los códices son el Códice Florentino de fray Bernardino de Sahagún y los tratados sobre plantas, animales y minerales de la Nueva España redactados por Francisco Hernández. Ambas obras recopilan datos obtenidos mediante una colaboración con

19 Se ha detectado también en los matices verdes del Códice Zouche-Nuttall, así como en el Códice Florentino (Baglioni et al. 2011: 100; Higgitt 2013). 
indígenas en la segunda mitad del siglo XVI. En el Códice Florentino, la información substancial sobre materiales colorantes se concentra en el capítulo 11 del libro XI, que ofrece descripciones en náhuatl de los elementos naturales utilizados para crear tintes, pigmentos y pigmentos laca en las épocas prehispánica y colonial temprana (Sahagún 1953-1982 XI: 239-245; Dupey 2015). La estructura de este capítulo es ilustrativa porque muestra de qué manera los nahuas categorizaban los colores en función de sus usos ${ }^{20}$.

Así, las materias colorantes orgánicas empleadas para fabricar los pigmentos laca aplicados en los códices se mencionan en los primeros dos apartados del capítulo, que cuenta cinco secciones en total. La primera de estas secciones se refiere a colorantes que si bien servían para teñir, eran primeramente pensados como materias para pintar los códices, en particular la grana cochinilla, el achiote, así como las flores de Cosmos y las especies de Cuscuta que servían para preparar pigmentos laca amarillos (supra). Las viñetas que ilustran esta sección, en efecto, muestran solamente a artistas pintando manuscritos (Sahagún 1979 III: 368r-370r). El título y las ilustraciones de la segunda sección del capítulo revelan que ésta reúne principalmente tintes y pigmentos laca, cuya fabricación requería que se fijara un colorante sobre un sustrato inerte, es decir, que seguía un método cercano a la tintura (Sahagún 1979 III: 370r-371v; Falcón 2014: 31-33). Esta sección aglutina el palo Brasil y el añil -que servían para teñir pero también para pintar códices-y los pigmentos laca texotli y tecozahuitl, que eran arcillas teñidas respectivamente de azul y de amarillo (supra).

La tercera sección del capítulo sobre colores de la obra de Sahagún, en cambio, trata sobre materias de origen mineral que servían para pintar, teñir o preparar pigmentos laca, por ejemplo el alumbre. Se describen también varios minerales blancos que se aplicaban sobre diferentes soportes, como objetos de cerámica, recipientes de jícaras y tecomates, o códices, donde fungían como base de preparación. Ciertos autores sostienen que uno de estos blancos, el tetizatl, así como el pigmento rojo llamado tlahuitl corresponden a la calcita $\left(\mathrm{CaCO}_{3}\right)$ y a la hematita que se han detectado en esculturas, murales y enlucidos del Templo Mayor de Tenochtitlan (López Luján et al. 2005: 17-19; Miriello et al. 2011: 1121). La cuarta sección del capítulo se dedica a colores obtenidos por mezcla -como los verdes yappalli y quiltic- al tiempo que regresa al tema de los pigmentos laca, explicando cómo se preparaban algunos a partir de sales metálicas, por ejemplo el rojo oscuro camopalli ${ }^{21}$. La quinta y última sección, finalmente, cita los principales términos del léxico cromático náhuatl (Dupey 2010: 271-277).

La estructura del capítulo sobre colores del Códice Florentino refleja la dicotomía que regía la categorización de los materiales colorantes en la sociedad náhuatl prehispánica: se presentan por separado las materias orgánicas que estaban en el origen de los pigmentos laca utilizados para pintar códices -algunas de las cuales se describen

20 Para otras interpretaciones acerca de la estructura de este capítulo y, en especial, sobre el uso de la Naturalis Historia de Plinio el Viejo como modelo de este texto y, más en general, del libro XI del Códice Florentino, véanse: Magaloni 2014:19-25; Falcón 2014: 19-21.

21 Diana Magaloni (2014: 21) nota acertadamente que los materiales colorantes que se presentan en esta cuarta sección del capítulo corresponden también a colores secundarios, como son el verde, el rojo oscuro o morado, y el café. 
con los tintes porque se empleaban también para teñir (secciones 1 y 2)- y los minerales que servían para colorear otros soportes (sección 3). Esto concuerda con los resultados de los análisis arqueométricos efectuados en esculturas y edificios erigidos en el Centro de México durante el Posclásico Tardío, pues si bien los códices fueron pintados con paletas complejas que incluían una multitud de pigmentos laca y un número reducido de minerales, las obras arquitectónicas y escultóricas, por el contrario, se colorearon sobre todo con minerales. Ejemplo de ello es el programa artístico del Templo Mayor de Tenochtitlan, que incluía esculturas, bajorrelieves y murales pintados con pigmentos, específicamente la calcita (blanco), la goetita $(\mathrm{FeO}(\mathrm{OH})$; amarillo) y la hematita (rojo) -a veces mezclada con magnetita $\left(\mathrm{Fe}_{3} \mathrm{O}_{4}\right)$ para obtener un rojo oscuro y brillante-, en tanto que únicamente los colores negro y azul se obtuvieron de materias orgánicas, el negro de humo y el índigo (López Luján et al. 2005: 17-27; López Luján y Chiari 2012: 335-336).

De la singular paleta cromática de los códices surge una pregunta sencilla a la que, sin embargo, no es fácil responder: ¿cuál fue el motivo que inspiró el desarrollo de una tecnología del color basada en materiales orgánicos para pintar los manuscritos nahuas prehispánicos? El problema que plantea esta pregunta parece aún más arduo de resolver porque los pigmentos blanco y rojo aplicados a las esculturas y estructuras arquitectónicas se derivan de minerales de fácil acceso para los antiguos nahuas. Los estudios de Miriello et al. (2011: 1121) han mostrado que la calcita empleada para los enlucidos blancos del Templo Mayor provenía de yacimientos cercanos a la antigua ciudad de Tula (Hidalgo), que se encontraba a unos 80 kilómetros de México Tenochtitlan. En cuanto a la hematita, López Luján et al. (2005: 18-19) señalan que si bien es delicado definir la procedencia de la que se utilizó en el Templo Mayor, se conocen «varias minas en las proximidades de Tenochtitlan, entre ellas las de la Sierra Patlachique en el Valle de Teotihuacan». Conviene agregar que una de las materias colorantes que los mercaderes del México Central vendían en Tabasco y Chiapas se llamaba tlahuitl (Sahagún 1953-1982 IX: 22) y correspondía al almagre (Molina 1970: 145r; Hernández 1959 III: 409), una arcilla coloreada por hematita, lo que confirma que los nahuas tenían un amplio acceso a este material, pues lo comercializaban.

Para preparar los pigmentos laca de sus códices, en cambio, los nahuas recurrían a menudo a plantas que no crecían naturalmente en el altiplano central mexicano. Los informantes de Sahagún (1953-1982 XI: 240-241; 2000: Libro XI, capítulo 11: 1130-1131; Dupey 2015: 232-233) y Hernández (1959 II: 27-28) coinciden en definir como zonas cálidas las regiones donde crecen el achiote, pero también las plantas con virtudes colorantes amarillas que servían para preparar el xochipalli y el zacatlaxcalli. Paralelamente, la grana cochinilla y el tecozahuitl eran productos que los nahuas conseguían, al menos en parte, a través del tributo que les entregaban ciudades de Guerrero y de la Mixteca Alta (Oaxaca) (Matrícula de Tributos 1980: folios 20, 23, 24; Códice Mendoza 1992: folios 40r, 43r, 44r, 45r). Entonces, a diferencia de los minerales, varias de las materias requeridas para fabricar los pigmentos laca de los códices no resultaban directamente accesibles - - , en el caso de los productos tributados, tal vez no eran accesibles en cantidades suficientes- en la región habitada por los nahuas del Posclásico. Estos datos añaden una interrogante suplementaria a la 
pregunta formulada precedentemente: ¿cuál fue el motivo que inspiró el desarrollo de una tecnología del color basada en materiales orgánicos, en parte de origen foráneo, para pintar los manuscritos nahuas prehispánicos?

Acerca de la singularidad de la paleta de los códices prehispánicos del México Central no se ha ofrecido una explicación detallada. Varios autores sostienen que era por su cualidad plástica que los pintores de manuscritos recurrían a pigmentos laca elaborados con materias orgánicas, pues éstos se caracterizan por una luminosidad que no poseen los materiales colorantes inorgánicos. Carolusa González Tirado (1998: 7; también Wallert 1995: 655; Magaloni 2011: 55, 2014: 38; Zetina et al. 2014: 137-138), por ejemplo, explica que:

«Si bien la obtención de los pigmentos de origen mineral era más sencilla a la vez que estos materiales se caracterizan por ser más resistentes al deterioro de los elementos exteriores, los tintes utilizados para preparar los pigmentos utilizados en los códices producen colores más vivos que las tierras coloreadas».

Este planteamiento coincide con lo que se observa en las obras. Los colores de la escultura monumental de Tenochtitlan se caracterizan por ser opacos (López Luján y Chiari 2012: 335). Los colores de los códices, en cambio, conservan su luminosidad, a pesar del paso del tiempo y de condiciones de conservación que no fueron siempre óptimas. De esta viveza se sorprendieron Sylvia Rodgers Albro y Thomas Albro (1990) al examinar el Códice de Huejotzingo, que fue exhibido en la Library of Congress de Washington de 1930 a 1942, pero cuya capa pictórica seguía brillante pues este manuscrito colonial temprano fue iluminado con pigmentos laca. Por mi parte, pude constatar lo mismo al examinar los códices Borgia, Cospi, Laud y Vaticano $B$ en sus repositorios europeos ${ }^{22}$, así como al observar algunas láminas del Códice Fejérváry-Mayer durante su exhibición en el World Museum de Liverpool.

Allende estas apreciaciones individuales, que el lector podrá juzgar demasiado subjetivas, los textos históricos confirman la preocupación de los antiguos nahuas por conseguir materias colorantes que confirieran luminosidad a la capa pictórica. En el capítulo sobre colores de la obra de Sahagún, los informantes del franciscano insisten sobre el carácter brillante del color obtenido de los pigmentos laca camopalli, xochipalli y yappalli:

«El camopalli. Es rojo oscuro, es algo para embellecer las cosas, es algo para dar luminosidad a las cosas, es algo para dar brillo a las cosas [...] (tlapalpoyahuac, tlacualnextiloni, tlacueponaltiloni, tlatlanexiotiloni).

El xochipalli. [...] Es un tinte, un pigmento, algo para embellecer las cosas, algo para dar luminosidad a las cosas, algo para dar resplandor a las cosas [...] (tlapaloni, tlacuiloloni, tlacualnextiloni, tlatonameyotiloni).

Yo doy luminosidad a las cosas con yappalli (iyappaltica nitlacueponaltia)» (Dupey 2015: 248, 258; también Sahagún 1953-1982 XI: 240, 244).

Conviene insistir sobre el hecho de que los materiales citados pertenecen al conjunto de colores para pintar los códices que se elaboraban a partir de materias or-

22 En la actualidad, los códices Borgia y Vaticano B se conservan en la Biblioteca Apostólica Vaticana, el Códice Cospi en la Biblioteca Universitaria di Bologna, y el Códice Laud en la Bodleian Library de Oxford. 
gánicas. Como vimos, el camopalli y el xochipalli eran pigmentos laca preparados precipitando respectivamente grana cochinilla y cosmos sobre sales metálicas; en tanto que el yappalli era un verde oscuro derivado de la mezcla de zacatlaxcalli con texotli, un término que nombraba los pigmentos laca obtenidos fijando tintes azules sobre arcillas (supra).

Estos datos históricos confirman lo que la observación de los códices revela acerca de su proceso de creación: la finalidad subyacente al desarrollo de una tecnología del color basada en materias orgánicas para pintar los manuscritos prehispánicos del México Central fue la obtención de una capa pictórica que se caracterizaba por su luminosidad. Además, los pasajes citados muestran que al utilizar una paleta basada en materias orgánicas, los pintores de códices garantizaban la belleza de sus obras. En efecto, la descripción de los pigmentos laca camopalli y xochipalli indica que la viveza de su color repercutía sobre la hermosura de las obras pintadas con ellos. Esto se confirma con la caracterización del tecozahuitl -un término que calificaba los pigmentos laca obtenidos fijando un tinte amarillo sobre una arcilla (supra)-como «algo para embellecer las cosas (tlacuauhnextiloni)» (Dupey 2015: 253; también Sahagún 1953-1982 XI: 242).

Adicionalmente, planteo que los colores brillantes convertían a los códices en obras estéticamente logradas dentro del canon imperante en el México Central durante el Posclásico Tardío, pues el rol vector de la belleza del color brillante se advierte no sólo en las descripciones de los colores usados para pintar manuscritos, sino también en discursos nahuas relativos a otras expresiones artísticas. Así, la descripción de la labor de los trabajadores de la pluma en el Códice Florentino insiste en la preocupación de estos artistas por crear la ilusión de la luz en sus obras, ya que expone cómo disponen sus materiales en capas de tal manera que generen reflejos luminosos (Sahagún 1953-1982 IX: 95-96; 10: 25), una cualidad plástica de las plumas especialmente apreciada y buscada por los antiguos nahuas (Russo 2011: 394-395). Esta obra se refiere también al trabajo de las piedras preciosas efectuado por los lapidarios:

«La desgastaban [la piedra], lisaban su superficie, y le daban un brillo metálico, y las abrillantaban con un trozo de madera, [...] con el cual la hacían perfecta (auh niman quichiqui, quisteca, ioan quitemetzhuia, ioan quipetlaoa itech quauitl [...]: inic quiiectilia)» (Sahagún 1953-1982 IX: 81).

Asimismo, es interesante que en el discurso del pintor acerca de su arte, este artista compara sus obras policromas con orejeras, es decir con joyas que en el México antiguo eran fabricadas, precisamente, con piedras preciosas pulidas:

«Yo pinto algo con una diversidad de colores, yo realizo diversos motivos en algo, yo embellezco algo, yo realizo algo con motivos complicados, multicolor como orejeras, lo realizo multicolor como orejeras, yo pinto algo (Ninepapantlacuiloa, nepapan tlacuiloli nicchihua, niccualnextia, tlamomoxoltic nicchihua, cuicuilchampotic, cuicuilchampochtic nicchihua, niquiccuiloa)» (Dupey 2015: 260; también Sahagún 1953-1982 XI:245).

Este pasaje reafirma que los colores participaban en el embellecimiento de las obras pictóricas y hacían que se parecieran a las joyas apreciadas por su brillo. 
Volviendo ahora a la discrepancia que advertimos inicialmente entre la paleta de colores luminosos y de origen orgánico usada en los manuscritos, por un lado, y los colores minerales y opacos empleados para la arquitectura y la escultura de Tenochtitlan, por el otro, parece razonable formular la hipótesis de que éstos últimos recibían un tratamiento para adquirir brillo y corresponder con los criterios estéticos en vigor en la sociedad náhuatl. Lo sugiere el que los pigmentos arcillosos, como la hematita y la goethita, empleados en pinturas murales de Teotihuacán o del área maya hayan sido bruñidos para garantizar su brillo (Magaloni 1995; Vázquez de Ágredos 2010). También lo expresa un fragmento de la obra de Sahagún que establece que, en su tiempo, los colores apagados de la arquitectura náhuatl pudieron ser brillantes y contribuir así a la creación de obras estéticamente superiores. El pasaje en cuestión se refiere a los palacios de los reyes, describiéndolos como construcciones embellecidas por sus colores y como estructuras arquitectónicas que «brillaban»:

«Tecpancalli. Esto significa la casa del rey o la casa de gobierno, allí es donde se encuentra el rey, allí es donde vive E....... No es un lugar común, es un lugar bueno, hermoso, es una cosa que ha sido embellecida ....... Es una casa pintada, es una casa pintada. Es magnífico, polícromo, cubierto de diseños complejos, de dibujos complicados. Brilla constantemente, es magnífico, es una maravilla (Tecpancalli: qujtoznequj, in tlatoanj ical anoço altepecalli, in vncan ca in vncan nemj tlatoanj, T...... a çan cana queçinamjcan çan quecinamjcan, qualli, iectli, tlacencaoalli ....... tlacuilolcalli, tlacujlolcali, maviztic, tlatlapalpoalli, tlamomoxoltic, moxtic, tôtônaticac, maviztic, tlamaviçolli)» (Sahagún 1953-1982 IX: 270-271).

\section{Conclusiones}

La materialidad del color en los códices se puede investigar comparando los resultados de los estudios arqueométricos y codicológicos efectuados en los manuscritos con los textos y las imágenes relativos a los colores y a su base material en la documentación del siglo XVI. Además, el examen físico de los códices, al igual que los discursos nativos acerca de las materias colorantes y de las técnicas artísticas, revela cuáles pudieron ser los fines estéticos subyacentes al uso del color en los códices pintados en el México Central durante el Postclásico. En efecto, la selección por parte de los pintores de pigmentos laca especialmente luminosos y su maestría en la aplicación de dichos materiales garantizaban la excelencia de los códices dentro del canon estético vigente en la cultura náhuatl prehispánica.

Como consecuencia, la paleta de color de los códices del México Central incluye principalmente pigmentos laca originados en materias colorantes orgánicas, algunas de las cuales han sido caracterizadas con técnicas arqueométricas, mientras que otras siguen sin identificar pero son descritas en las fuentes históricas. En cuanto al uso de pigmentos se observa que, por un lado, quedaba restringido a lo estrictamente necesario: en concreto, se recurría a minerales blancos para elaborar la «base de preparación» de los códices que servía de soporte a la capa pictórica. Por otro lado, la presencia del oropimente en la gama cromática de ciertos códices confirma el interés de los artistas por conseguir colores luminosos pues, a diferencia del carácter opaco 
de muchos pigmentos, este trisulfuro de arsénico es un material amarillo sumamente brillante.

Lo que lleva a concluir que entre toda la información histórico-cultural que aportan las obras de arte prehispánico es particularmente significativo el testimonio que nos dan acerca de su proceso de creación y de las intenciones de sus creadores.

\section{Referencias bibliográficas}

Álvarez ICAZA Longoria, María Isabel

2014 El Códice Laud, su tradición, su escuela y sus artistas. Tesis de doctorado en Historia del Arte: México: Universidad Nacional Autónoma de México.

Baglioni, Piero, Rodorico Giorgi, Marcia Carolina Arroyo, David Chelazzi, Francesca Ridi y Diana MAGALONI KERPEL

2011 «On the Nature of the Pigments of the General History of the Things of New Spain: The Florentine Codex», en Colors between Two Worlds. The Florentine Codex of Bernardino de Sahagún, Gerhard Wolf, Joseph Connors y Louis A. Waldman, eds, pp. 78-105. Florencia: Max-Planck-Institute, Villa I Tatti.

Batalla Rosado, Juan José

2008a El Códice Borgia. Una guía para un viaje alucinante por el inframundo. Madrid: Biblioteca Apostólica Vaticana / Testimonio Compañía Editorial S.A.

2008 b «Los códices mesoamericanos: métodos de estudio», Itinerarios. Revista de Estudios lingüísticos, literarios, históricos y Antropológicos 8: 43-65.

Boone, Elizabeth $\mathrm{H}$.

1990 «The Painting Styles of the Manuscripts of the Borgia Group», en Circumpacifica: Festschrift für Thomas S. Barthel, Bruno Illius y Matthias Laubscher, eds., vol. 1, pp. 35-54. Frankfurt am Main: Peter Lang.

2007 Cycles of Time and Meaning in the Mexican Books of Fate. Austin: University of Texas Press.

Buti, David, Davide Domenici, Costanza Miliani, Concepción García, Teresa Gómez, Félix Jímenez, Ana Verde, Ana Sabía, Aldo Romani, Federica Presciutti, Brenda Doherty, Bruno G. Brunetti y Antonio Sgamellotti

2014 «Non-invasive Investigation of a Pre-Hispanic Maya Screenfold Book: the Madrid Codex». Journal of Archaeological Science 42: 166-178.

CAso, Alfonso

1927 «Las ruinas de Tizatlán». Revista Mexicana de Estudios Históricos 1(4): 139-172.

Códice Mendoza

1992 The Codex Mendoza, 4 vols., edición de Frances F. Berdan y Patricia Anawalt. Berkeley: University of California Press.

Contreras Martínez, José Eduardo

1994 «Las estructuras polícromas de Ocotelulco, Tlaxcala. Arquitectura, historia y descripción», en Calendario de pronósticos y ofrendas. Libro explicativo del llamado Códice Cospi, Ferdinand Anders, Maarten Jansen y Peter Van Der Loo, eds., pp. 109-117. México: Fondo de Cultura Económica. 
DARK, Philip y Joyce Plesters

1959 «The palimpsests of Codex Selden: Recent Attempts to Reveal the Covered Pictographs», en Actas del XXXIII Congreso Internacional de Americanistas, v. 2, pp. 530-539. San José (Costa Rica): Lehmann.

Doménech, Antonio, María Teresa Doménech, Cristina Vidal, María Luisa VÁzQuez, Laura Osete, Francisco M. Valle

2014 «Discovery of indigoid-containing clay pellets from La Blanca: significance with regard to the preparation and use of Maya Blue». Journal of Archaeological Science 41: 147-155.

Domenici, Davide, David Buti, Costanza Miliani, Bruno Brunetti y Antonio SGamellotti

2014 «The Colours of Indigenous Memory: Non-invasive Analyses of Pre-Hispanic Mesoamerican Codices», en Science and Art: The Painting Surface, Antonio Sgamellotti, Bruno Brunetti y Costanza Miliani, eds., pp. 94-119. Cambridge: Royal Society of Chemistry.

DuPEY GARCÍA, Élodie

2010 Les couleurs dans les pratiques et les représentations des Nahuas du Mexique central (XIVe-XVI siècles). Tesis de doctorado en Historia de las Religiones y Antropología Religiosa. París: École Pratique des Hautes Études.

2015 «Traducción del náhuatl al español del capítulo once del libro XI del Códice florentino.» Estudios de Cultura Náhuatl 49: 239-265.

Dupey García, Élodie y María Isabel Álvarez ICAZA Longoria

En prensa «El color en los códices del llamado Grupo Borgia: diferencias y convergencias», en Estilo y región en el arte mesoamericano, Pablo Escalante Gonzalbo y María Isabel Álvarez Icaza Longoria, eds. México: Universidad Nacional Autónoma de México, Instituto de Investigaciones Estéticas.

Escalante Gonzalbo, Pablo

1996 El trazo, el cuerpo y el gesto. Los códices mesoamericanos y su transformación en el valle de México en el siglo XVI. Un análisis del cambio histórico en el arte de la pictografía, con especial dedicación al problema de la representación del cuerpo humano, sus formas, sus posturas y sus ademanes. Tesis de doctorado en Historia. México: Universidad Nacional Autónoma de México.

2010 Los códices mesoamericanos antes y después de la conquista española. Historia de un lenguaje pictográfico. México: Fondo de Cultura Económica.

FALCón Álvarez, Tatiana

2014 Tintes de otoño. Experimentación con plantas tintóreas para la reinterpretación de los saberes, tradiciones y usos del color en manuscritos indígenas. Tesis de maestría en Historia del Arte. México: Universidad Nacional Autónoma de México.

GonzÁlez TIRAdo, Rocío Carolusa

1998 Análisis de pigmentos en ocho códices mexicanos sobre piel. Tesis de maestría en Ciencia de la Conservación. Leicester: De Montfort University.

HaUde, Mary Elizabeth

1998 «Identification of Colorants on Maps from the Early Colonial Period of New Spain (Mexico)». Journal of the American Institute for Conservation 37 (3): 240-270.

HERNÁNDEZ, Francisco

1959 Obras completas. Universidad Nacional Autónoma de México, Instituto de Investigaciones Históricas. 
1998 De Materia Medica Novae Hispaniae. Libri Quatuor. Cuatro libros sobre la materia médica de Nueva España. El Manuscrito de Recchi. Madrid, Valladolid: Ediciones Doce Calles, Junta de Castilla y León.

HigGitT, Catherine

2013 Materials and Techniques of the Pre-Columbian Mixtec Manuscript, the Codex Zouche-Nuttall, MOLAB User Report. Londres: British Museum. Documento en línea: http://www.eu-artech.org/files/Users_report_final_NUTTAL.pdf.

LANDA, Diego de

1994 Relación de las cosas de Yucatán. México: Consejo Nacional para la Cultura y las Artes.

Laurencich Minelli, Laura, Giorgio Gasparotto y Giovanni Valdrè

1993 «Notes about the Painting Techniques and the Morphological, Chemical and Structural Characterization of the Writing Surface of the Prehispanic Mexican Codex Cospi». Journal de la Société des Américanistes 79 (1): 203-207.

López Luján, Leonardo, Giacomo Chiari, Alfredo LóPez Austin y Fernando CARrizosa 2005 «Línea y color en Tenochtitlan. Escultura policromada y pintura mural en el recinto sagrado de la capital mexica». Estudios de Cultura Náhuatl 36: 15-45.

LóPEz Luján, Leonardo y Giacomo CHIARI

2012 «Color in monumental Mexica sculpture». Res. Anthropology and Aesthetics 61/62: 330-342.

Magaloni Kerpel, Diana

1995 «El espacio pictórico teotihuacano, tradición y técnica», en La pintura mural prehispánica en México I, Teotihuacán, Beatriz De La Fuente, ed., vol. 2, pp. 187225. México: Universidad Nacional Autónoma de México, Instituto de Investigaciones Estéticas.

2011 «Painters of the New World. The Process of Making the Florentine Codex», en Colors Between Two Worlds. The Florentine Codex of Bernardino de Sahagún, Gerhard Wolf, Joseph Connors y Louis A. Waldman, eds., pp. 46-76. Florencia: Max-Planck-Institute, Villa I Tatti.

2014 The Colors of the New World. Artists, Materials, and the Creation of the Florentine Codex. Los Ángeles: Getty Publications.

Maldonado Alvarado, Mauricio y Benjamín Maldonado Alvarado

2004 La sabiduría de las pieles. De las técnicas de curtición de los códices a la curtiduría tradicional actual en Oaxaca. Oaxaca: Instituto de Investigaciones en $\mathrm{Hu}-$ manidades de la Universidad Autónoma Benito Juárez de Oaxaca, Secretaría de Asuntos Indígenas del Gobierno del Estado, Instituto Nacional de Antropología e Historia.

Matrícula de Tributos

1980 Edición facsímil, con comentarios de Frances Berdan y Jacqueline de DurandForest. Graz: Academische Druck und Verlagsanstalt.

Miliani, Costanza, Davide Domenici, Catia Clementi, Federica Presciutti, Francesca Rosi, David Buti, Aldo Romani, Laura Laurencich Minelli y Antonio Sgamellotti

2012 «Colouring materials of pre-Columbian codices: non-invasive in situ spectroscopic analysis of the Codex Cospi». Journal of Archaeological Science 39: 672-679. 
Miriello, Domenico, Donatella Barca, Gino M. Crisci, Luis Barba, Jorge Blancas, Agustín Ortíz, Alessandra PeCCI y Leonardo LóPez Luján

2011 «Characterization and Provenance of Lime Plasters from the Templo Mayor of Tenochtitlan (Mexico City)». Archaeometry 53 (6): 1119-1141.

Molina, Alonso de

1970 Vocabulario en lengua castellana y mexicana y mexicana y castellana. México: Porrúa.

Motolinía (Toribio de Benavente)

1971 Memoriales o Libro de las cosas de la Nueva España y de los naturales de ella. México: Universidad Nacional Autónoma de México, Instituto de Investigaciones Históricas.

Olmos, Andrés de

2004 Le vocabulaire de l'Arte de la lengua mexicana de Andrés de Olmos, Sybille de Pury y Marc Thouvenot, eds. Documento en línea: http://www.sup-infor.com

Reyes VALERIo, Constantino

1993 De Bonampak al Templo Mayor. El azul maya en Mesoamérica. México: Siglo Veintiuno.

Rodgers Albro, Sylvia y Thomas C. Albro

1990 «The Examination and Conservation Treatment of the Library of Congress Harkness 1531 Huejotzingo Codex». Journal of the American Institute for Conservation 29 (2): 97-115.

Roquero, Ana

2006 Tintes y tintoreros de América. Catálogo de materias primas y registro etnográfico de México, Centro América, Andes Centrales y Selva Amazónica. Madrid: Ministerio de Cultura, Secretaría General Técnica.

Russo, Alessandra

2011 «Uncatchable Colors», en Colors Between Two Worlds. The Florentine Codex of Bernardino de Sahagún, Gerhard Wolf, Joseph Connors y Louis A. Waldman, eds., pp. 388-410. Florencia: Max-Planck-Institute, Villa I Tatti.

SAHAGun, Bernardino de

1953-82 Florentine Codex. General History of the Things of New Spain, 12 vols. Santa Fe: University of Utah, School of American Research.

1979 Códice Florentino. Manuscrito 218-20 de la Colección Palatina de la Biblioteca Medicea Laurenziana, 3 vols. México: Secretaría de Gobernación, Archivo General de la Nación.

2000 Historia general de las cosas de Nueva España, 3 vols. México: Consejo Nacional para la Cultura y las Artes.

SELER, Eduard

1990 «Codex Borgia and Allied Aztec Picture Writing», en Collected Works in Mesoamerican Linguistics and Archaeology, John Erick S. Thompson y Francis B. Richardson, eds., v. 1, pp. 39-44. Culver City (California): Labyrinthos.

1963 Códice Borgia, 3 vols. México, Buenos Aires: Fondo de Cultura Económica. 
STREBINGER, Robert

1961 «Mikroanalystische Untersuchung der Farbstoffe des Codex Philipp J. Becker I», en Codices Becker I/II, Karl Anton Nowotny, ed., p. 23. Graz: Akademische Druck-Und Verlagsanstalt.

TORRES, Luis, Antonio SotomaYor y Ticul Álvarez

1966 «Análisis de los materiales del Códice», en Interpretación del Códice Colombino, Antonio Caso y Mary Elizabeth Smith, eds., pp. 89-102. México: Sociedad Mexicana de Antropología.

Torres Montes, Luis Alejandrino

2001 «Dyes and pigments», en The Oxford Encyclopedia of Mesoamerican Cultures, David Carrasco, ed., vol. 1, pp. 346-348. Oxford: Oxford University Press.

VÁzQuez de Ágredos Pascual, María Luisa

2010 La pintura mural maya. Materiales y técnicas artísticas. Mérida: Universidad Nacional Autónoma de México.

WALLERT, Arie

1995 «On Some Natural Organic Yellow Colorants in Aztec Codices: The Florentine Codex», en Materials Research Society Symposium Proceedings, v. 352. Issues in Art and Archaeology IV, P. B. Vandiver, J. R. Druzik, J. L. Galvan, I. C. Freestone, G. Segan, eds., pp. 653-662. Pittsburg: Materials Research Society.

Zetina, Sandra, José Luis Ruvalcaba, Tatiana Falcón, Eumelia Hernández, Carolusa GonzÁlez, Elsa Arroyo y Marimin LóPEz CÁCERES

2008 «Painting Syncretism: A Non-Destructive Analysis of the Badiano Codex», en 9th International Conference on NDT of Art, Jerusalem Israel, 25-30 May 2008. Documento en línea: http://www.ndt.net/search/docs.php3?MainSource=65.

Zetina, Sandra, José Luis Ruvalcaba, Marimin López Cáceres, Tatiana Falcón, Eumelia Hernández, Carolusa González y Elsa Arroyo

2011 «Non Destructive In Situ Study of Mexican Codices: Methodology and First Results of Materials Analysis for the Colombino and Azoyu Codices», en Proceedings of the 37th International Symposium on Archaeometry, Isabella TurbantiMemmi, ed., pp. 349-354. Springer-Verlag Berlin Heidelberg.

Zetina, Sandra, José Luis Ruvalcaba, Tatiana Falcón, Jesús Arenas Alatorre, Saeko Yanagisawa, Marisa Álvarez ICAZa Longoria y Eumelia Hernández

2014 «Material Study of the Codex Colombino», en Science and Art. The Painting Surface, Antonio Sgamellotti, Bruno G. Brunetti y Costanza Miliani, eds., pp. 120146. Londres: Royal Society of Chemistry. 